\title{
Optimal Design of Track Hanging Structure Based on Order Algorithm
}

\author{
Hao Qin \\ Shanghai Institute of Special Equipment Inspection and Technical Research, Shanghai 200333, China; \\ School of Mechanical Engineering, Shanghai Jiao Tong University, Shanghai 200052, China \\ XiaoYing Tang, WenQing Yao \& FengQi Wu \\ Shanghai Institute of Special Equipment Inspection and Technical Research, Shanghai 200333, China
}

\begin{abstract}
Based on mechanical analysis of main beam structure in track hanging, the design optimization problem of weight of the girder structure was transformed into solution of optimal parameters values under mechanical constraints optimal parameters. Adopting structure geometry value as input parameters, structural strength, stiffness, stability as response parameters, a mathematical optimization model was established based on the order algorithm. Optimization results of the main beam were obtained by finite element numerical calculation. The results show that this new design method can reduce the weight of the main beam, improve the efficient use of materials.
\end{abstract}

KEYWORD: order algorithm; FEM analysis; crane structure; optimal design

\section{INTRODUCTION}

The higher safety coefficient, which is frequently used in the design of the construction machinery track structure, causes low material use efficiency and needless waste. Box girder is very common structure part of the track crane, thus the optimization for the girder structure can guarantee the safety and decrease the weight of the structure simultaneously. However, due to the complicated working condition and the geometrical and nonlinear characteristic of the structure, the passive verification method of strength and stiffness are normally concerned (Du Xuewu, 2005). The structure optimization, replacing the passive check method by initiative designing the structure scheme and initial section size, can obtain the best result from all of the possible schemes (Fei Jinggao, 2002) (Guo Tong, 2007).

Through analyzing the structure and load feature of track crane girder, the gravity, inertia force and wind loading are considered to establish the optimal design model of the girder. The girder maximum or minimum problem under structure stiffness, strength and stability constraint condition could be transformed into extremum problem under no constraint condition, and then the optimal design result of the girder geometrical parameters are obtained. The corresponding results can provide valuable references to sufficiently utilize the bearing capability of the components and control the structure weight.

\section{STRUCTURE CHARACTERISTICS OF THE TRACK CRANE GIRDER}

As the primary bearing component, girder sustains the working load directly and has high demands for strength, stability and least possible weight. Boxtype double bridge frame, easily manufactured and highly optimized for general use, is the widely used structure type. Whereas it also has some drawbacks such as high weight, easy bend of the girder and low horizontal stiffness (Guo Tong, 2007) (He Fei, 2009). Consequently, it is essential to optimally design the girder to decrease the structure weight and meet the use requirement simultaneously.

The external load of girder mainly includes deadweight, inertia force and wind load (Han Yanli, 2011). Dead weight could be calculated as follow:

$F=m g$

Where $m$ is the mass of the component and $g$ is the acceleration of gravity.

Inertia force is calculated by the mechanical acceleration of the additional motion:

$F=m a$

Where $a$ is the acceleration of the component.

Wind load $p_{1}$ is obtained according to standard (Li Weizhong, 2013):

$$
p_{1}=0.625 \mathrm{v}_{s}^{2}
$$


Where $\mathrm{V}_{S}$ is the wind speed, $\mathrm{A}$ is the windward area and $\varphi$ is the angle between component orientation and wind direction.

\section{OPTIMAL DESIGN METHOD OF THE TRACK CRANE GIRDER}

\subsection{Mechanical model for optimal design of the track crane girder structure}

In this paper, the girder overall strength, stiffness and stability are used as the constraint function and geometrical size parameter as the design variables, then the optimal girder geometrical size could be obtained by the multi-variable optimal analysis. Ultimately, the girder weight decreases to improve material use efficiency. The corresponding section model of track crane girder is shown in Fig. 1 and Fgi. 2, of which the design variables could be indicated by a set of mutual independent parameters:

$$
\begin{aligned}
& X=\left[\begin{array}{llllll}
H & B & T_{1} & T_{2} & T_{3} & T_{4}
\end{array}\right]^{T} \\
& =\left[\begin{array}{llllll}
X_{1} & X_{2} & X_{3} & X_{4} & X_{5} & X_{6}
\end{array}\right]^{T}
\end{aligned}
$$

Where $H$ and $B$ are the girder height and width, respectively; $T_{1}$ and $T_{2}$ are the width of the top and bottom plate; $T_{3}$ and $T_{4}$ are the left and right web plate thickness.

In the case of fixed girder span, weight of girder is proportional to sectional area. Consequently, the sectional area is conformed as the aim function.

$S=\left(T_{1}+T_{2}\right) B+\left(T_{3}+T_{4}\right) H$

$=\left(X_{3}+X_{4}\right) X_{2}+\left(X_{5}+X_{6}\right) X_{1}$

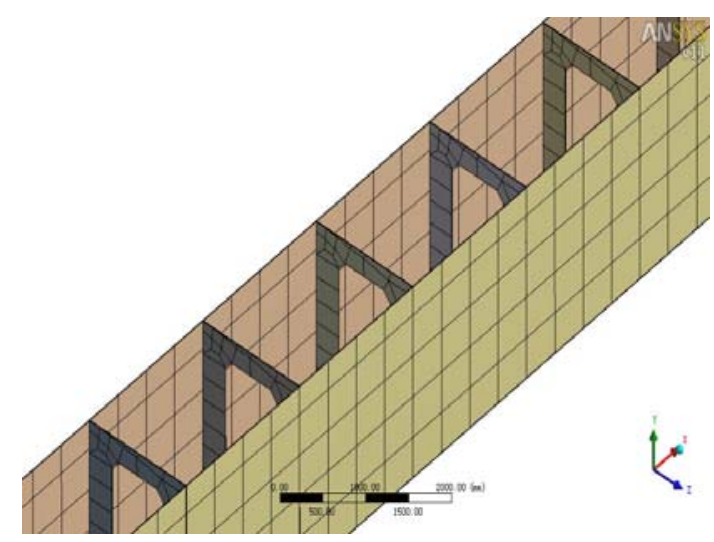

Fig.1. Cutaway view of the partial mesh

According to the track crane design standard (He Fei, 2009), the constraint condition of girder is that the maximum stress should be less than the allowable stress:

$$
\sigma_{\max } \leq[\sigma]
$$

Where $[\sigma]$ is the allowable stress of the structure

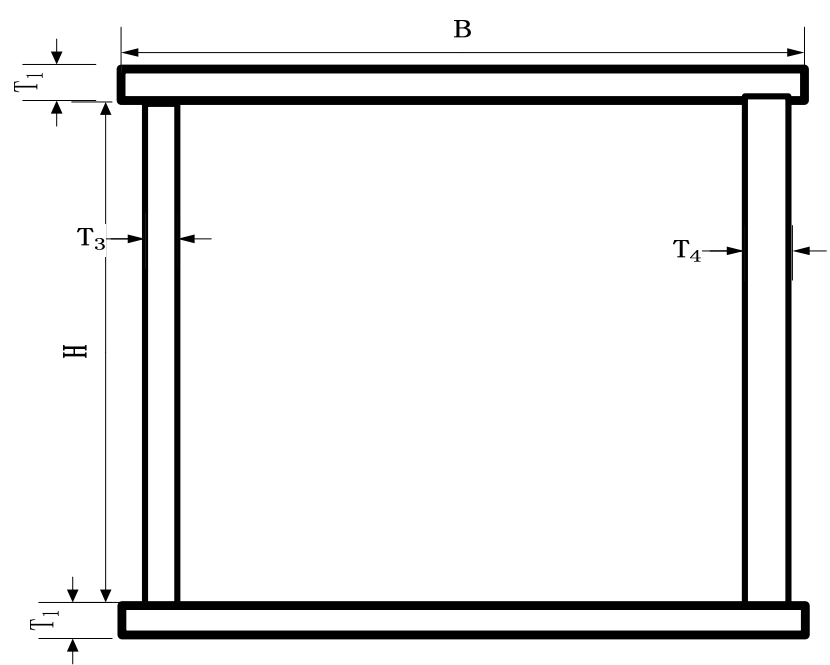

Fig.2. Box girder sectional view

The constraint condition of the girder stiffness is that the deflection in the middle of the girder $f \leq\left(\frac{1}{700} L \sim \frac{1}{1000} L\right)(L$ is the girder span). Here $f \leq \frac{1}{800} L$ is determined as the optimal design constraint condition of the girder.

$$
f \leq \frac{1}{800} L
$$

The girder stability constraint condition is that the optimized buckling eigenvalue should be less than original one, namely,

$$
\lambda \leq \lambda_{0}
$$

where, $\lambda_{0}$ is the buckling eigenvalue before optimizing.

Combining the aim function and constraint condition, the equation set we could obtain is as follow:

$$
\left\{\begin{array}{l}
\min S=\left(t_{1}+t_{2}\right) b+\left(t_{3}+t_{4}\right) h \\
\sigma_{\max } \leq[\sigma] \\
f \leq \frac{1}{800} L \\
\lambda \leq \lambda_{0}
\end{array}\right.
$$

From the solution of the equation (9), the engineering problem can be converted to the optimal solution problem under mechanical constraint condition. 


\subsection{Optimal method based on the order algorithm}

In this paper, the order algorithm (Qin Dongchen et al, 2010) (Tao Yuanfang et al, 2012) (Yu Lanfeng, 1996) (Zhao Xiaowei et al, 2012) is employed to solve the optimal solution problem under above mechanical constraint conditions. Based on the sampling statistical analysis, the order algorithm could find the optimal solution by fitting the response function of the design variable, state variable and aim function.

In the minimum problem presented by equation (9), design variable could be determined as follows:

$\underline{x}_{i} \leq x_{i} \leq \bar{x}_{i}$

Where $\underline{x}_{i}, \bar{x}_{i}$ is the upper and lower boundary of $X_{i}$.

The equation (11) and (12) could be selected as the constraint condition and aim function, respectively.

$$
\begin{aligned}
& \left\{\begin{array}{l}
\hat{\sigma}(X)=\sigma(X)+\varepsilon_{g} \\
\hat{f}(X)=f(X)+\varepsilon_{h} \\
\hat{\lambda}(X)=\lambda(X)+\varepsilon_{w}
\end{array}\right. \\
& \hat{S}(X)=S(X)+\varepsilon_{f}
\end{aligned}
$$

Where $\varepsilon$ is a small parameter.

The square fitting method is usually method in engineering problems, thus the fitting equation of equation (11) and (12) are as follows:

$\hat{S}=a_{0}+\sum_{i}^{n} a_{i} x_{i}+\sum_{i}^{n} \sum_{j}^{n} b_{i j} a_{i} x_{j}$

On the basis of equation (11) to (13), constraint minimum problem could be rewritten in following form:

$$
\left\{\begin{array}{l}
\min \hat{S}=\hat{S}(X) \\
\underline{x}_{i} \leq x_{i} \leq \bar{x}_{i}(i=1,2,3, \ldots, 6) \\
\hat{\sigma}(X) \leq \bar{\sigma}_{i}+\alpha_{i}\left(i=1,2,3, \ldots, m_{1}\right) \\
\hat{f}(X) \leq \bar{f}_{i}+\beta_{i}\left(i=1,2,3, \ldots, m_{2}\right) \\
\hat{\lambda}(X) \leq \bar{\lambda}_{i}+\delta_{i}\left(i=1,2,3, \ldots, m_{3}\right)
\end{array}\right.
$$

Where $\bar{\sigma}_{i}, \bar{f}_{i}, \bar{\lambda}_{i}$ are the state variable and $\alpha_{i} 、 \beta_{i} 、 \delta_{i}$ represent the allowance of the state variable.

The Lagrange equation (Zhang Xiaoli, 2007) of extremal problems under constraint condition is used to modify the equation (14):

$$
\begin{aligned}
F\left(x, p_{k}\right)=\hat{S} & +f_{0} p_{k}\left(\sum_{i=1}^{m_{1}} X\left(x_{i}\right)+\sum_{i=1}^{m_{2}} G\left(\hat{\sigma}_{i}\right)\right. \\
& \left.+\sum_{i=1}^{m_{2}} H\left(\hat{f}_{i}\right)+\sum_{i=1}^{m_{3}} W\left(\hat{\lambda}_{i}\right)\right)
\end{aligned}
$$

where, $x_{i}$ is the design variable and $f_{0}$ is the reference value of the aim function; $p_{k}$ is the response surface parameter, and $X$ is the penalty function for imposing on the variable constraint, while $G, H, W$ are the penalty functions of the state variable constraint. The penalty function is shown in equation (16).

$X\left(x_{i}\right)= \begin{cases}\frac{c_{1}+c_{2}}{x^{u}-x_{i}}, & x_{i}<x^{u}-\varepsilon\left(x^{u}-x_{i}\right) \\ \frac{c_{3}+c_{4}}{x_{i}-x^{l}}, & x_{i} \geq x^{u}-\varepsilon\left(x^{u}-x_{i}\right)\end{cases}$

$(i=1,2,3, \ldots n)$

Where $x^{u}, x^{l}$ are the upper and lower boundary of the design variable respectively, while $c_{1}, c_{2}, c_{3}, c_{4}$ is coefficient and $\varepsilon$ is small parameter.

Transforming the constraint condition and aim function to the unrestraint equation through equation (14) to (16), the optimal solution of the unrestraint minimum aim function could be obtained by finite element method.

\section{RESULT AND DISCUSSION}

The finite element method is used to calculate design variable values of the extremal problem for corresponding track crane girder structure; and the calculation results are shown in Table 1 to 2 .

Table 1 Structural calculation results

\begin{tabular}{llll}
\hline \multirow{2}{*}{ value } & stress & deformation & \multirow{2}{*}{ Bucking } \\
\cline { 2 - 3 } & $\mathrm{MPa}$ & $\mathrm{mm}$ & 1.660 \\
Original & 105.1 & 18.654 & 1.5873 \\
Optimization & 110.4 & 19.725 & $4.37 \%$ \\
ratio & $4.96 \%$ & $-5.74 \%$ & \\
\hline
\end{tabular}

Table 1 shows the maximum stress, maximum deformation and buckling eigenvalue of the original and deformed girder structure. It is can be seen from Table 1 that in the original and optimized girder structure, the positions of maximum equivalent stress are both in the constraint region of the outrigger, and differ only by $4.96 \%$. The maximum displacement deformation of the original and optimized girder structure present at middle of the bottom flange plate, differing by $5.74 \%$. Both the maximum 
equivalent stress and displacement of optimized girder structure meet the requirement of design standard. The bucking eigenvalue of optimized girder structure reduces, indicating that overall and local stability is better than before.

Table 2 Geometrical size of girder structure

\begin{tabular}{llllll}
\hline \multirow{2}{*}{ value } & $\begin{array}{l}\text { Top } \\
\text { plate }\end{array}$ & $\begin{array}{l}\text { Bottom } \\
\text { plate }\end{array}$ & height & width & weight \\
\cline { 2 - 6 } & $\mathrm{mm}$ & $\mathrm{mm}$ & $\mathrm{mm}$ & $\mathrm{mm}$ & $\mathrm{t}$ \\
\hline Original & 14 & 12 & 2300 & 1500 & 47.58 \\
Optimization & 13 & 11 & 2100 & 1400 & 37.93 \\
ratio & $7.1 \%$ & $8.3 \%$ & $8.7 \%$ & $6.7 \%$ & $20.3 \%$ \\
\hline
\end{tabular}

Table 2 gives the geometrical parameter calculation results of the optimized structure. The geometrical parameters of the optimized components decrease the structure weight under the constraints condition of the strength, stiffness and stability. The thickness of top and bottom plate, height and width of girder decrease by approximately 7 8\%, and the weight of girder decrease by $20.3 \%$.

\section{CONCLUSION}

In this paper, the extremal problem under mechanical constraint condition is transformed to unconditional extremal problem by order algorithm, and the geometrical parameters of track crane girder are optimized to decrease the weight of the girder by $20 \%$. The main conclusions are as follows:

(1) Through the optimal calculation, the weight of girder structure decreases by approximately 20.28\% when the strength, stiffness and stability meet the requirements. Compared with the original structure, the thickness plate, width and height of the girder decrease by approximately $8 \%$, which improves the material use ratio.

(2) Based on the mechanical model, FEM calculation and optimal design, the bearing capability of the structure components can be rationally utilized to control the structure weight, to achieve lean manufacturing and promote the beneficial efficiency in enterprises on the premise of meeting structure safety and rationally design.

\section{REFERENCES}

Crane design handbook edit group. crane design handbook. China machine press. 1980.

Du Xuewu. Augmented Lagrangian Function Methods for Solving Constrained Optimization Problems. Shanghai University, 2005.

Fei Jinggao. A zero order algorithm real-time systematicly controlled by differential-algebra. Progress in Natural Science, 2002, 02: 105-108.
Guo Tong. Application Comparison Between Zero-Order And First-Order Optim Ization Methods In Model Updating Of Suspension Bridges. Journal of Vibration and Shock, 2007, 04:35-38+68+167-168.

Guo Tong. Research in form-finding of suspension structures based on newton-raphson iteration and zero order optimization arithmetic. Engineering Mechanics, 2007, 04: 142$146+158$.

Han Yanli. Research on penalty function of constrained optimization problems. Institutes of Technology of Henan, 2011.

He Fei. Force analysis and research for the bridge crane girder. Science and Technology Innovation Herald, 2009, 17-45.

Li Weizhong. Grider structure optimization of the doubletrolley crane. Hoisting and Conveying Machinery, 2013, 11 : 9-13.

Qin Dongchen, Yan Lili. Structural optimization design of box girder of bridge crane. Mining \& Processing, 2010, 12: 4851.

Tao Yuanfang, Shi Xiaofei. Optimization Design for Crane girder based on improved particle swarm algorithm. Chinese Journal of Construction Machinery. 2012, 01: 50-53.

Yu Lanfeng. Optimal design for the box girder of single girder overhead crane. Mechanical science and technology, 1996, 02: 198-202.

Zhang Xiaoli. Topological optimization design for corrugatedweb structure of overhead traveling crane. Journal of the Chongqing institute of technology, 2007, 04:30-32.

Zhao Xiaowei, Wei Liangbao. Fast Optimization Design for the Bridge Crane Based on APDL. Journal of Taiyuan University of Science and Technology, 2012, 01: 49-53. 